\title{
Changing Clinical Pattern of Dengue Fever and its Unusual Manifestations- 2019 Outbreak in Dhaka, Bangladesh
}

\author{
QT ISLAM ${ }^{\mathrm{a}}$, HB SAGOR ${ }^{\mathrm{b}}, \mathrm{TC}^{\mathrm{T}} \mathrm{TULI}^{\mathrm{c}}, \mathrm{FM}^{\mathrm{NOOR}}{ }^{\mathrm{d}}$, MM ISLAM $^{\mathrm{e}}$
}

\begin{abstract}
:
Background: In 2019 Bangladesh faced largest outbreak of Dengue fever (DF) and clinicians observed some changing patterns in presentation and laboratory findings of DF. This study intends to find the changing patterns of dengue and its different manifestations including unusual one.
\end{abstract}

Material and Methods: This study deals around 220 dengue patients visited to Popular Medical College Hospital as inpatient of medicine department during the period 1st July 2019 to 31st December 2019. Chi-square statistics was used find the intra and inter association among the disease manifestations and multinomial logistic regression analysis was used to compare disease severity.

Results: Among 220 dengue serologically positive cases, the most common age group affected was 18-49 years (68.6\%). Regarding the known common features like

Introduction:

Dengue fever (DF) is a highly endemic infectious disease of the tropical countries and is rapidly becoming a global burden. Globally, almost $50 \%$ of the population is estimated to be under risk resulting

a. Dr. Quazi Tarikul Islam, Professor of Medicine, Popular Medical College, Dhaka-1205, Bangladesh.

b. Hironmoy Barman Sagor, Assistant Registrar, Department of Medicine, Popular Medical College, Dhaka-1205, Bangladesh.

c. Tasmina Chowdhury Tuli, Registrar, Department of Medicine, Popular Medical College, Dhaka-1205, Bangladesh.

d. Farha Musharrat Noor, Statistician, Department of Statistics, Dhaka University, Dhaka-1000, Bangladesh.

e. Md. Momin Islam, Statistician, Department of Statistics, Dhaka University, Dhaka-1000, Bangladesh.

Adddress of Correspondence: Dr. Quazi Tarikul Islam, Affiliation Prof. of Medicine, Popular Medical College, H \# 16, R \# 2, Dhanmondi, Dhaka-1205, Phone no : 01715299399, Email : prof.tarik@gmail.com

Received: 27 October, 2019

Accepted: 22 November, 2020 fever (91.8\%), vomiting (50.2\%), headache (48.2\%), were frequent among the patients but myalgia (19.6\%), rash (14.5\%), retro orbital pain (14.1\%) were not so common. Presence of unusual features like abdominal pain (49.3\%), loose motion (55\%) and cough/ breathlessness (41.4\%) ascites (53.2\%), pleural effusion (51.8\%), hepatomegaly (32.3\%), peri-gallbladder edema (35.5\%) and splenomegaly (15.1\%) were also common among dengue patients. Serum Lipase was also increased in (28.8\%) cases suggesting pancreatitis.

Conclusion: Unusual Manifestations of dengue fever is common. As the spread of dengue is increasing with significant morbidity and mortality, it is extremely important for the clinicians to know about its unusual manifestations.

Keywords: Unusual manifestations; Diarrhoea; Breathlessness; Splenomegaly; Pancreatitis.

(J Bangladesh Coll Phys Surg 2021; 39: 9-18)

DOI: https://doi.org/10.3329/jbcps.v39i1.50457

in 390 million new dengue infections in each year ${ }^{1}$. In the recent years, the incidence of dengue is increasing in developing countries with increased mortality. New onset of dengue cases in a dengue free area, clustering of cases, cases with atypical manifestations have made DF mandatory to get included under notifiable diseases.

Dengue is a tropical disease affected by single stranded RNA flavivirus spread by the bite of female Aedes aegypti and Aedes albopictus mosquitoes ${ }^{3}$.The virus has four sero-types namely, DEN-1, DEN-2, DEN-3 and DEN-4 4 . Infection with one sero-type gives lifelong immunity to same sero-type and temporary immunity to other sero-types ${ }^{4}$. Infection with multiple sero-types and secondary infections manifest severely than primary infections ${ }^{5}$. Dengue fever is usually a non-specific and self-limiting biphasic febrile illness. Clinical presentation of Dengue fever varies with a wide spectrum of signs

and symptoms. Infection can be asymptomatic or cause a range of severity from mild dengue fever to 
dengue hemorrhagic fever, which can then progress to dengue shock syndrome and death ${ }^{6}$. Atypical manifestations are rare and include encephalopathy, encephalitis, seizures, hepatocellular damage, ARDS, acute renal failure, acalculous cholecystitis, myocarditis, pericardial effusion, severe gastrointestinal hemorrhage, Guillain-Barre syndrome and rhabomyolysis ${ }^{7,8}$.

Bangladesh first experienced a large outbreak of Dengue in the year 2000 with 5551 cases and case fatality was 93. The containment of the disease was successfully handled afterward and was rewarding. Even between the year 2007 to 2010 there were very lower number of cases reported with no death record. From 2015 the incidence of cases started rising with few death This is obviously a change in epidemiology of the disease. Over 10,000 people, primarily in Dhaka, had been infected with the mosquito-borne disease in 2018. But strikingly in 2019 Bangladesh faced a nationwide outbreak affecting all districts ${ }^{2}$ that began primarily in in April. According to Director General of Health Services (DGHS), 101354 patients were affected and 179 died in 2019.With rising disease burden, atypical manifestations also noticed in many of the cases which are missed most often due to lack of awareness. Our aim of the study was to look for current patterns of the presentation along with unusual manifestations.

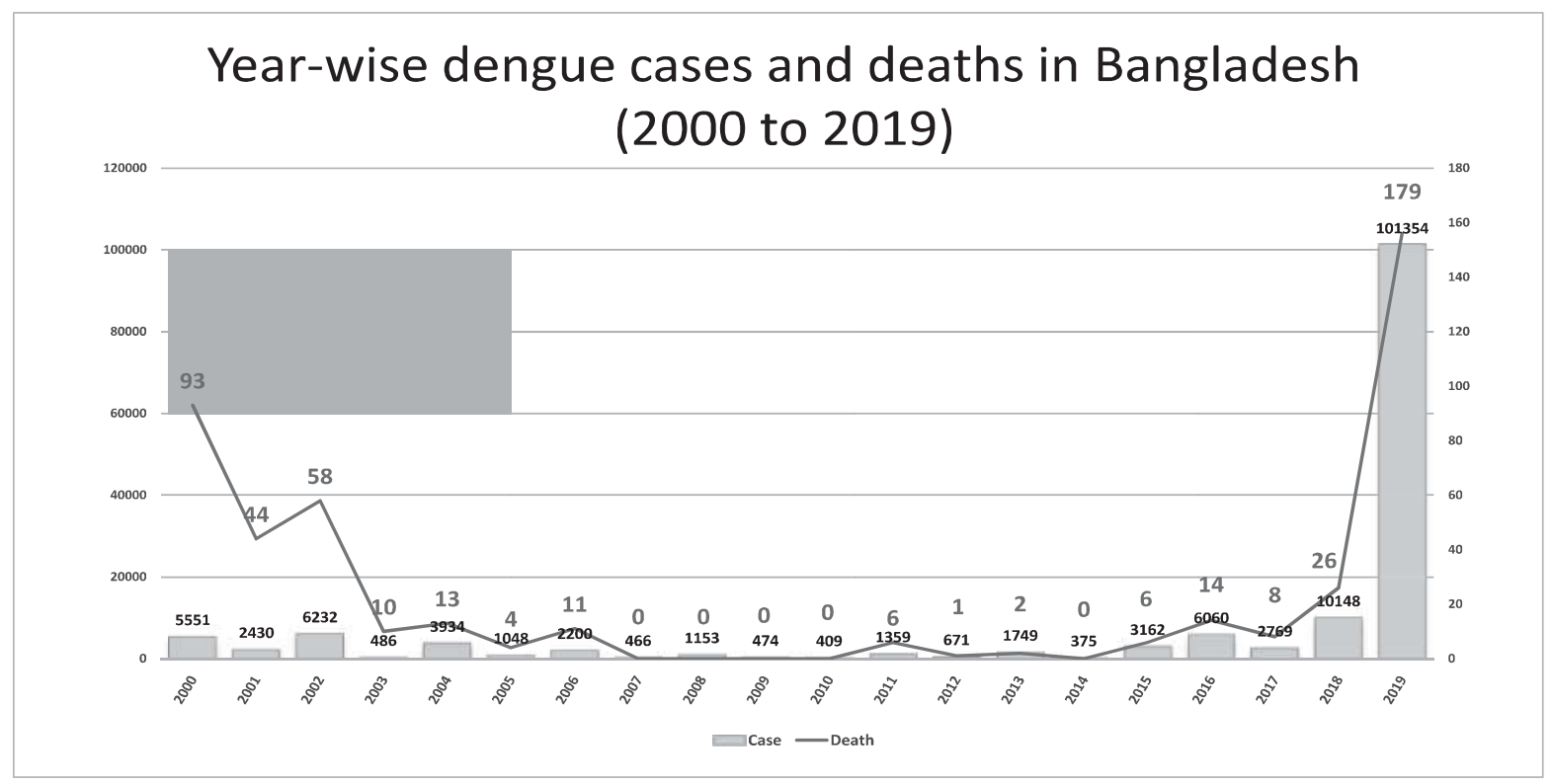

Source: Disease Control Room, DGHS, Mohakhali, Dhaka, January 2020

According to National Guideline for Clinical Management of Dengue Syndrome -2018, Dengue Case classified by severity into 3 groups ${ }^{-}$

i) Group-A: (Home Care): Patient who don't have a warning sign.

ii) Group-B (Hospital Care): Patient with warning signs.

iii) Group C: (must be hospitalized) Patient with any of the following features:

- Severe plasma leakage with shock or/and fluid accumulation with resp. distress

- $\quad$ Severe bleeding

- Severe organ impairment

- Severe metabolic dysfunction

Symptoms apart from the mentioned features are often co-existing. Many health care providers had expressed the need for expanding the list of atypical symptoms under typical dengue. Study aimed to search the varieties of symptoms of dengue and its unusual manifestations in 2019 outbreak. 


\section{Materials and Methods:}

\section{Ethics statement}

Informed written consent had been taken from patients. They had the freedom to withdraw from the study at any time, if they desired.

It had been assured that all information and records would be kept confidential and the procedures would be helpful for both the physician and the patients in making rational approach to the care management. Privacy of the patients had been strictly maintained.

\section{Study setting and design}

This is a descriptive observational, record-based study done in the Department of Medicine, Popular Medical College and Hospital, Dhaka during July 1, 2019 to December 31, 2019. Total of 220 dengue patient's record who had dengue fever (DF) both clinical and lab confirmed - either by nonstructural protein1 (NS1) antigen positive or anti-dengue immunoglobulin $\mathrm{M}(\mathrm{Ig} \mathrm{M})$ antibody positive during this study period were included in this study. Dengue with comorbid conditions that may affect the outcome such as diabetes, hypertension, Pregnancy, menstruation was also included. Data had been entered in a standard proforma (Case Record Form) prepared by literature review and expert opinion. Dengue infection was classified according to National Guideline for Clinical Management of Dengue Syndrome -2018.

A detailed clinical history, physical examination and baseline investigations were undertaken and followed up till the patient got discharged from hospital. All clinical and laboratory details were carefully reviewed with daily assessment during hospital stay as per pre-tested questionnaire. The study includes demographic variables including age, sex and clinical diagnoss variables namely febrile diarrhea, abdominal pain, dyspnea, vomiting, pleural effusion, ascites, hepatomegaly, splenomegaly, gall bladder edema, rash, abdominal pain and investigational variables mainly ALT, AST. Out of above clinical diagnoses, unusual manifestations of dengue such as febrile diarrhea, gall bladder edema, abdominal pain, hepatomegaly, splenomegaly and increased lipase are considered for further study.

\section{Statistical analysis}

Data collected from the dengue patients were analyzed using Stata version 16. Categorical variables were expressed as frequencies (percentages) and chi-square test was performed to find the significance of those variables. The patient's data were tabulated according to categorical variables including sex, clinical manifestations, comorbidity, radiological finding of DF, diagnostic and biochemical laboratory finding of DF. Continuous variables such as age, days of illness, SGOT (AST), SGPT (ALT), and lipase were also categorized. Any variable with $p \leq 0.05$ on chi-square analysis was considered statistically significant and then further analyzed in the multinomial logistic regression analysis to compare group $\mathrm{B}$ and group $\mathrm{C}$ relative to group $\mathrm{A}$ for severe clinical manifestations of dengue patients. This data was used to find the relationship between demography, clinical manifestations and laboratory parameters with severity of dengue patients. The analyses were performed at $95 \%$ confidence interval with $\mathrm{p}<0.05$.

\section{Result:}

\section{Baseline characteristics of dengue patients}

Among total 227 cases, 220 confirmed cases were evaluated. Due to insufficient data 7 cases were dropped from study. Among 220 confirmed cases, $118(53.6 \%)$ were male and $102(46.4 \%)$ were female. Majority of the cases $151(68.6 \%)$ were from the age group of 18 to 49 years old. Age group $<18$ years and age group 50 years and above were found $32(14.6 \%)$ and 37 (16.8\%) respectively.

(Table 1).

Most common clinical features were found as fever 202(91.8\%), vomiting $110(50.2 \%)$, headache 106 (48.2\%), myalgia 43 (19.6\%), abdominal pain 108 $(49.3 \%)$, rash $32(14.5 \%)$, retro orbital pain 31 (14.1\%), hypotension 29 (13.2\%), low pulse pressure $36(16.4 \%)$. Others symptoms included loose motion 121 (55\%), cough/ breathlessness 91 (41.4\%), bleeding $27(12.3 \%)$, bradycardia $12(5.5 \%)$ were also present. Comorbidities were reported among 76 patients including hypertension 27 (12.3\%), diabetes $31(14.1 \%)$, pregnancy $8(3.6 \%)$ and menstruation 10 (4.6\%).Most of the patient were Dengue NS1 positive $202(91.8 \%)$ but about $18(8.2 \%)$ cases were Dengue NS1 negative which were tested within 5 days of febrile episodes and later confirmed by Dengue -IgM positive test. Among 220 cases about $90(40.9 \%)$ cases were dengue-IgG positive and 78 (35.5\%) were dengue-IgM positive. Ascites and pleural effusion 
Table 1: Demographic characteristics and distribution of dengue fever using the data set from Popular Medical College and Hospital during the period July 1, 2019 to December 31, 2019.

\begin{tabular}{|c|c|c|c|c|c|}
\hline \multirow[t]{3}{*}{ Characteristics } & \multirow{2}{*}{$\begin{array}{c}\text { Total patients } \\
\mathbf{N}=\mathbf{2 2 0}\end{array}$} & \multicolumn{3}{|c|}{ Distribution of dengue fever } & \multirow[t]{3}{*}{ P-value } \\
\hline & & Group A (72) & Group B (111) & Group C (37) & \\
\hline & $\mathbf{N}(\%)$ & $\mathbf{N}(\%)$ & $\mathbf{N}(\%)$ & $\mathbf{N}(\%)$ & \\
\hline Sex & & & & & \multirow[b]{3}{*}{0.003} \\
\hline Male & 118 (53.6) & $41(34.8)$ & 49 (41.5) & $28(23.7)$ & \\
\hline Female & $102(46.4)$ & $31(30.4)$ & $62(60.8)$ & $9(8.8)$ & \\
\hline Age & & & & & \multirow{4}{*}{0.038} \\
\hline$<18$ & $32(14.6)$ & $13(40.6)$ & $11(34.4)$ & $8(25.0)$ & \\
\hline $18-49$ & $151(68.6)$ & $53(35.1)$ & $74(49.0)$ & $24(15.9)$ & \\
\hline 50 or above & $37(16.8)$ & $6(16.2)$ & $26(70.3)$ & $5(13.5)$ & \\
\hline Days of illness & & & & $1(50.0)$ & \multirow{8}{*}{0.301} \\
\hline Day 0 & $2(0.9)$ & $1(50.0)$ & $0(0.0)$ & $2(33.3)$ & \\
\hline Day 2 & $6(2.7)$ & $1(16.7)$ & $3(50.0)$ & $3(12.5)$ & \\
\hline Day 3 & $24(10.9)$ & $6(25.0)$ & $15(62.5)$ & $10(20.0)$ & \\
\hline Day 4 & $50(22.7)$ & $16(32.0)$ & $24(48.0)$ & $14(20.6)$ & \\
\hline Day 5 & $68(30.9)$ & $18(26.5)$ & $36(52.9)$ & $4(7.8)$ & \\
\hline Day 6 & $51(23.2)$ & $20(39.2)$ & $27(52.9)$ & $3(15.8)$ & \\
\hline Day $7+$ & $19(8.6)$ & $10(52.6)$ & $6(31.6)$ & & \\
\hline
\end{tabular}

were very frequent on radiology report, which were respectively $117(53.2 \%)$ and $114(51.8 \%)$. Besides, hepatomegaly $71(32.3 \%)$, splenomegaly $33(15.1 \%)$ and gallbladder edema $78(35.5 \%)$ were also common among dengue patients. Alanine transaminase $($ ALT) $<100 \mathrm{U} / \mathrm{L}$ and ALT within $100-500 \mathrm{U} / \mathrm{L}$ were found in a larger proportion of patients $114(51.8 \%)$ and $79(35.9 \%)$ respectively compared to Aspartate transaminase(AST) $<100 \mathrm{U} / \mathrm{L}$ and AST within 100-500U/L 75 (34.1\%) and 69 (31.4\%) respectively. But, AST $>1000 \mathrm{U} / \mathrm{L}$ and AST within $500-1000 \mathrm{U} / \mathrm{L}$ were found in higher proportion of patients $32(14.5 \%)$ and $44(20.0 \%)$ respectively compared to ALT> $1000 \mathrm{U} / \mathrm{L}$ and ALT within 500-1000U/L $11(5.0 \%)$ and 16 (7.3\%). Lipase was increased in 63(28.8\%) cases (Table 2).

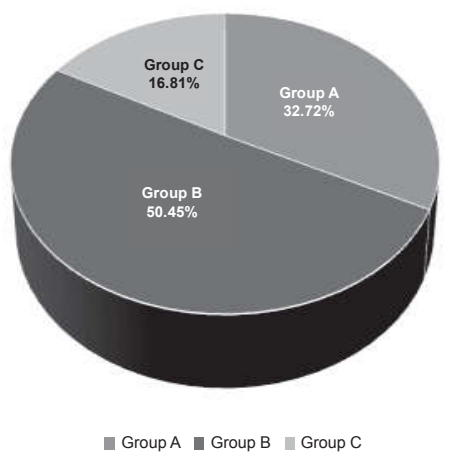

Figure 1: Distribution of dengue cases by group of severity among study population.
Findings by classification of groups according to "National Guideline for Clinical Management of Dengue Syndrome -2018”

Total 220 dengue patients were classified according to "National Guideline for Clinical Management of Dengue Syndrome -2018", by severity into 3 groups as: Group A (low), Group B (mild/moderate) and Group $\mathrm{C}$ (severe). The total number of patients in group B were found higher 111 (50.45\%) compared to group A 72 (32.72\%) and group C 37 (16.81\%) (Figure 1). A significant relationship was detected between gender and dengue classification groups ( $\mathrm{p}=$ 0.003). Dengue infections were observed more frequently in group B both male $49(41.5 \%)$ and female $62(60.8 \%)$ individuals. Notably, male patients more frequently developed severe dengue 28 (23.7\%) compared to female patients 9 (8.8\%) (Figure 2).A significant relationship was detected between age group and dengue classification groups $(p=0.038)$ at $5 \%$ level of significance. Dengue infection was observed more frequently in age group $<18$ years who were classified into group $\mathrm{A}(40.6 \%)$. Most of the patients belong to group B at the age group 18- 49 years $74(49.0 \%)$ and age group 50 and above 26(70.3\%) (Table 1). 
Table 2: Clinical characteristics and distribution of dengue fever using the data set from Popular Medical College and Hospital during the period July 1, 2019 to December 31, 2019.

\begin{tabular}{|c|c|c|c|c|c|}
\hline \multirow{3}{*}{ Characteristics } & \multirow{3}{*}{$\begin{array}{c}\text { Total } \\
\text { patients } \\
(220)\end{array}$} & \multicolumn{3}{|c|}{ Distribution of dengue fever } & \multirow[b]{3}{*}{ P-value } \\
\hline & & Group A (72) & Group B (111) & Group C (37) & \\
\hline & & $\mathrm{N}(\%)$ & $\mathrm{N}(\%)$ & $\mathrm{N}(\%)$ & \\
\hline \multicolumn{6}{|l|}{ Clinical manifestation } \\
\hline Fever & $202(91.8)$ & $59(29.2)$ & $107(53.0)$ & $36(17.8)$ & 0.001 \\
\hline Vomiting & $110(50.2)$ & $22(20.0)$ & $59(53.6)$ & $29(26.4)$ & $<0.001$ \\
\hline Headache & $106(48.2)$ & $38(35.8)$ & $55(51.9)$ & $13(12.3)$ & 0.200 \\
\hline Loose motion & $121(55)$ & $18(14.9)$ & $73(60.3)$ & $30(24.8)$ & $<0.001$ \\
\hline Pain abdomen & $108(49.3)$ & $20(18.5)$ & $54(50.0)$ & $34(31.5)$ & $<0.001$ \\
\hline Cough & $91(41.4)$ & $12(13.2)$ & $55(60.4)$ & $24(26.4)$ & $<0.001$ \\
\hline Rash & $32(14.5)$ & $3(9.4)$ & $14(43.8)$ & $15(46.9)$ & $<0.001$ \\
\hline Bleeding & $27(12.3)$ & $2(7.4)$ & $8(29.6)$ & $17(63.0)$ & $<0.001$ \\
\hline Retro-orbital pain & $31(14.1)$ & $13(41.9)$ & $13(41.9)$ & $5(16.2)$ & 0.481 \\
\hline Hypotension & $29(13.2)$ & $1(3.4)$ & $4(13.8)$ & $24(82.8)$ & $<0.001$ \\
\hline Bradycardia & $12(5.5)$ & $2(16.7)$ & $4(33.3)$ & $6(50.0)$ & 0.007 \\
\hline Low pulse pressure & $36(16.4)$ & $8(22.2)$ & $9(25.0)$ & $19(52.8)$ & $<0.001$ \\
\hline Myalgia & $43(19.6)$ & $17(39.5)$ & $21(48.8)$ & $5(11.7)$ & 0.440 \\
\hline \multicolumn{6}{|l|}{ Co-morbidity } \\
\hline Hypertension & $27(12.3)$ & $3(11.1)$ & $20(74.1)$ & $4(14.8)$ & 0.020 \\
\hline Diabetes & $31(14.1)$ & $3(9.7)$ & $24(77.4)$ & $4(12.9)$ & 0.003 \\
\hline Pregnancy & $8(3.6)$ & $0(0.0)$ & $7(87.5)$ & $1(12.5)$ & 0.079 \\
\hline Menstruation & $10(4.6)$ & $0(0.0)$ & $7(70.0)$ & $3(30.0)$ & 0.122 \\
\hline \multicolumn{6}{|c|}{ Radiological finding of $D F$} \\
\hline Hepatomegaly & $71(32.3)$ & $1(1.4)$ & $46(64.8)$ & $24(33.8)$ & $<0.001$ \\
\hline Splenomegaly & $33(15.1)$ & $1(3.0)$ & $9(27.3)$ & $23(69.7)$ & $<0.001$ \\
\hline Gallbladder edema & $78(35.5)$ & $3(3.85)$ & $50(64.1)$ & $25(32.0)$ & $<0.001$ \\
\hline Ascites & $117(53.2)$ & $5(4.3)$ & $81(69.2)$ & $31(26.5)$ & $<0.001$ \\
\hline Pleural effusion & $114(51.8)$ & $3(2.6)$ & $80(70.2)$ & $31(27.2)$ & $<0.001$ \\
\hline \multicolumn{6}{|c|}{ Diagnostic and biochemical laboratory finding of $D F$} \\
\hline NS1 positive & $202(91.8)$ & $66(32.7)$ & $101(50.0)$ & $35(17.3)$ & 0.785 \\
\hline Dengue Ig M Positive & $78(35.5)$ & $21(26.9)$ & $44(56.4)$ & $13(16.7)$ & 0.351 \\
\hline Dengue Ig G Positive & $90(40.9)$ & $16(17.8)$ & $54(60.0)$ & $20(22.2)$ & $<0.001$ \\
\hline \multicolumn{6}{|l|}{$S G O T(A S T)$} \\
\hline $\operatorname{High}(<100 \mathrm{U} / \mathrm{L})$ & $75(34.1)$ & $57(76.0)$ & $15(20.0)$ & $3(4.0)$ & \\
\hline $100-500 \mathrm{U} / \mathrm{L}$ & $69(31.4)$ & $13(18.8)$ & $53(76.8)$ & $3(4.4)$ & $<0.001$ \\
\hline $500-1000 \mathrm{U} / \mathrm{L}$ & $44(20.0)$ & $1(2.3)$ & $36(81.8)$ & $7(15.9)$ & \\
\hline$>1000 \mathrm{U} / \mathrm{L}$ & $32(14.5)$ & $1(3.1)$ & $7(21.9)$ & $24(75.0)$ & \\
\hline \multicolumn{6}{|l|}{$S G P T(A L T)$} \\
\hline $\operatorname{High}(<100 \mathrm{U} / \mathrm{L})$ & $114(51.8)$ & $69(60.9)$ & $39(33.9)$ & $6(5.2)$ & \\
\hline $100-500 \mathrm{U} / \mathrm{L}$ & $79(35.9)$ & $1(1.3)$ & $68(86.1)$ & $10(12.7)$ & $<0.001$ \\
\hline $500-1000 \mathrm{U} / \mathrm{L}$ & $16(7.3)$ & $1(6.2)$ & $3(18.8)$ & $12(75.0)$ & \\
\hline$>1000 \mathrm{U} / \mathrm{L}$ & $11(5.0)$ & $1(9.1)$ & $1(9.1)$ & $9(81.2)$ & \\
\hline \multicolumn{6}{|l|}{ Lipase } \\
\hline $\operatorname{High}(>300 \mathrm{U} / \mathrm{l}$ & $63(28.8)$ & $2(3.2)$ & $32(50.8)$ & $29(46.0)$ & $<0.001$ \\
\hline
\end{tabular}


Clinical manifestations of dengue infection demonstrated that patients classified with group B were more frequently present with fever $(\mathrm{p}=0.001)$, vomiting $(\mathrm{p}<0.001)$, loose motion $(\mathrm{p}<0.001)$, abdominal pain $(\mathrm{p}<0.001)$, cough $(\mathrm{p}<0.001)$, headache $(\mathrm{p}=0.200)$, retro-orbital pain $(\mathrm{p}=0.481)$, myalgia $(p=0.440)$. Clinical manifestations of dengue patients classified with Group-C were more frequently present with rash $(\mathrm{p}<0.001)$, bleeding $(\mathrm{p}<$ $0.001)$, hypotension $(\mathrm{p}<0.001)$, bradycardia $(\mathrm{p}=$ $0.007)$, low pulse pressure $(\mathrm{p}<0.001)$. A significant relationship was found between diabetes $(p=0.003)$ and hypertension $(\mathrm{p}=0.020)$ with dengue classification group. Those were highly occurred in group B. Relatively high proportion of group B dengue infected patients suffered from hepatomegaly $(\mathrm{p}<0.001)$, gallbladder edema $(\mathrm{p}<0.001)$, ascites $(\mathrm{p}$ $<0.001)$, pleural effusion $(\mathrm{p}<0.001)$ and high proportion of group $\mathrm{C}$ patients suffered from splenomegaly $(\mathrm{p}<0.001)$. A significant result was found between dengue IgG positive and dengue classification group ( $\mathrm{p}<0.001)$. IgG positive result was observed more frequently in group B 54 (60\%). In spite of giving insignificant result, most of the group B patients were observed giving Dengue IgM positive $44(56.4 \%)$ and NS1 positive result 101 (50.0\%).A significant result was found between dengue classification group and aspartate transaminase $(\mathrm{AST}) \quad(\mathrm{p} \quad<0.001)$ and alanine transaminase $($ ALT) $(p<0.001)$. A high proportion of patients with $\mathrm{AST}<100 \mathrm{U} / \mathrm{L}$ was found in group A, AST (100-500 U/L) and AST (500-1000 U/L) were found highly in group B and AST $(>1000 \mathrm{U} / \mathrm{L})$ was found highly in group C. Also for ALT, a high proportion of patients with ALT $<100 \mathrm{U} / \mathrm{L}$ was found in group A, ALT (100-500 U/L) was found highly in group B and ALT (500-1000 U/L) and ALT $(>1000 \mathrm{U} / \mathrm{L})$ were found highly in group C. A significant result was found between dengue group and lipase $(>300 \mathrm{U} / \mathrm{L})(\mathrm{p}<0.001)$ and a high proportion of patients were found in group B (Table 2).

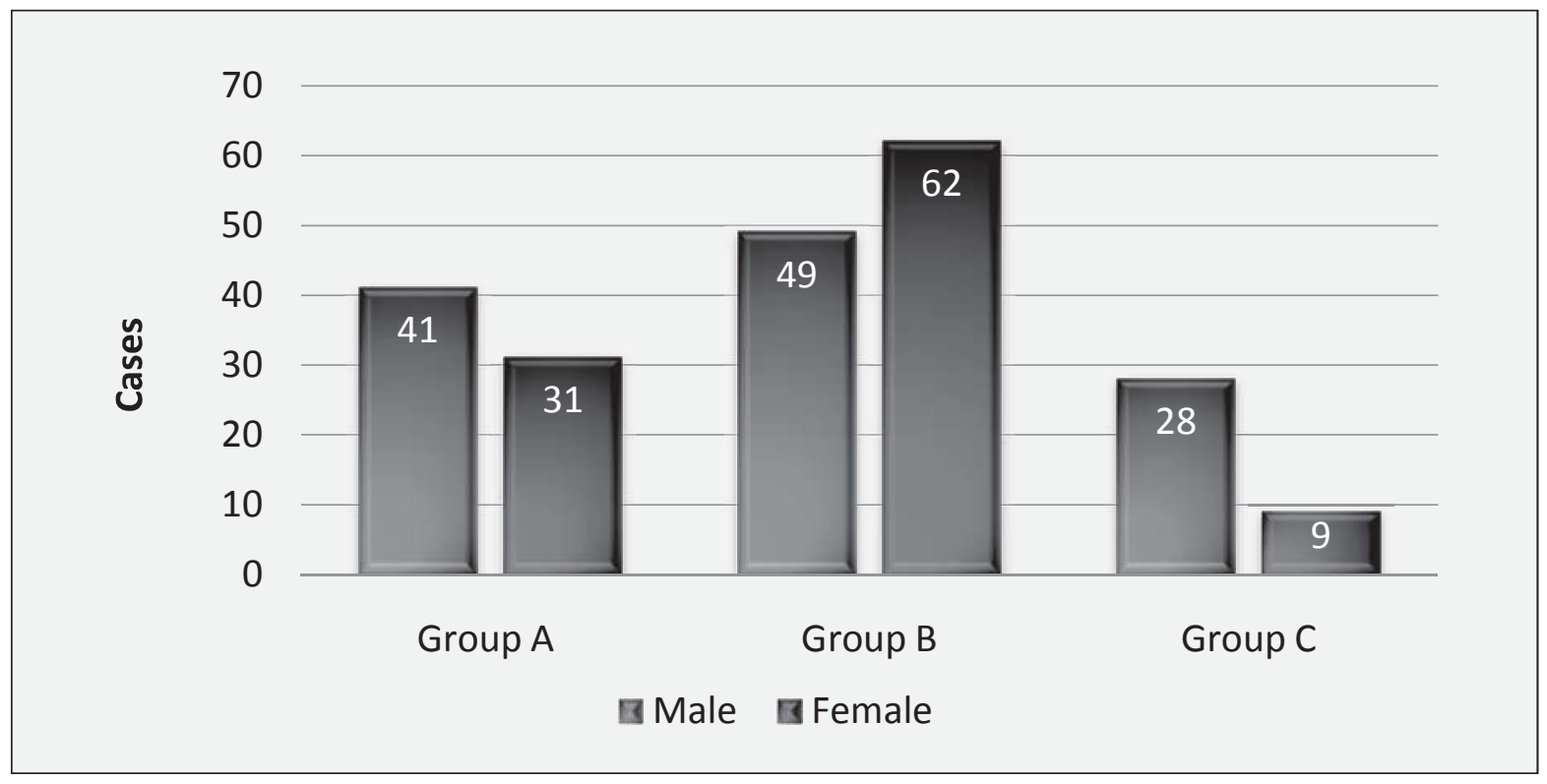

Figure 2: Gender differentiation with Group of Dengue cases.

\section{Multinomial logistic regression model}

Table 3 summarized the multinomial logistic relative odds ratio (ROR) estimate of group B and group C relative to group A dengue patients.

\section{Comparison of group B relative to group A}

The relative odds of being in group $B$ rather than in group A for having cough was significantly 4.25 (ROR: 4.25, 95\%CI: (1.02, 17.73); p value $=0.047$ ) times or $(4.25-1)^{*} 100 \%=325 \%$ higher than the corresponding relative odds for not having cough keeping all other covariates at fixed level.

The relative odds of being in group B rather than in group A for having rash was significantly 12.14 (ROR:12.14, 95\% CI: $(1.62,91.28)$; $\mathrm{p}$ value $=0.015)$ times or $(12.14-1) * 100 \%=1114 \%$ higher than the corresponding relative odds for not having rash keeping all other covariates at fixed level. 
Table 3: Multinomial logistic regression model using the data set from Popular Medical College and Hospital during the period July 1, 2019 to December 31, 2019.

\begin{tabular}{|c|c|c|c|c|}
\hline \multirow[t]{2}{*}{ Characteristics } & \multicolumn{2}{|c|}{ Group B } & \multicolumn{2}{|l|}{ Group C } \\
\hline & ROR (95\% CI) & P value & ROR (95\% CI) & P value \\
\hline \multicolumn{5}{|c|}{ Group A (Base Outcome) } \\
\hline $\begin{array}{c}\text { Gender } \\
\text { Female } \\
\end{array}$ & $1.43(0.41,5.11)$ & 0.574 & $1.003(0.02,39.05)$ & 0.10 \\
\hline $\begin{array}{l}\text { Age group } \\
18-49 \\
50 \text { or above }\end{array}$ & $\begin{array}{l}1.32(0.18,9.52) \\
2.45(0.17,35.4) \\
\end{array}$ & $\begin{array}{l}0.779 \\
0.509\end{array}$ & $\begin{array}{l}0.03(3.2 \mathrm{e}-05, \quad 23.55) \\
0.02(7.49 \mathrm{e}-07, \quad 457.2)\end{array}$ & $\begin{array}{l}0.297 \\
0.439\end{array}$ \\
\hline $\begin{array}{c}\text { Fever } \\
\text { Yes }\end{array}$ & $3.75(0.29,47.21)$ & 0.306 & $0.04(4.87 \mathrm{e}-07, \quad 4332.9)$ & 0.598 \\
\hline $\begin{array}{c}\text { Vomiting } \\
\text { Yes }\end{array}$ & $1.33(0.34,5.21)$ & 0.673 & $58.61(0.14,23747.7)$ & 0.184 \\
\hline $\begin{array}{c}\text { Loose motion } \\
\text { Yes }\end{array}$ & $3.18(0.89,11.35)$ & 0.074 & $0.89(0.01,77.01)$ & 0.962 \\
\hline $\begin{array}{l}\text { Pain Abdomen } \\
\text { Yes }\end{array}$ & $0.84(0.19,3.53)$ & 0.808 & $166.95(0.27,103069.1)$ & 0.119 \\
\hline $\begin{array}{l}\text { Cough } \\
\text { Yes }\end{array}$ & $4.25(1.02,17.73)$ & 0.047 & $30.72(0.56,1669.94)$ & 0.093 \\
\hline $\begin{array}{l}\text { Rash } \\
\text { Yes }\end{array}$ & $12.14(1.62,91.28)$ & 0.015 & $359.41(1.98,65072.0)$ & 0.027 \\
\hline $\begin{array}{r}\text { Bleeding } \\
\text { Yes }\end{array}$ & $1.55(0.10,22.62)$ & 0.750 & $135.52(0.11,161279.6)$ & 0.174 \\
\hline $\begin{array}{c}\text { Hypotension } \\
\text { Yes } \\
\end{array}$ & $3.99(0.01,1278.1)$ & 0.638 & $627.88(0.51,771766.7)$ & 0.076 \\
\hline $\begin{array}{c}\text { Bradycardia } \\
\text { Yes } \\
\end{array}$ & $2.42(0.07,86.32)$ & 0.627 & $31118.2(2.04,4.75 \mathrm{e}+08)$ & 0.035 \\
\hline $\begin{array}{l}\text { Low pulse pressure } \\
\text { Yes }\end{array}$ & $0.55(0.07,3.96)$ & 0.553 & $8.93(0.24,338.67)$ & 0.238 \\
\hline $\begin{array}{c}\text { Hypertension } \\
\text { Yes }\end{array}$ & $1.84(0.10,33.41)$ & 0.679 & $0.42 \quad(5.7 \mathrm{e}-06,30836.4)$ & 0.879 \\
\hline $\begin{array}{r}\text { Diabetes } \\
\text { Yes } \\
\end{array}$ & $3.22(0.22,47.14)$ & 0.393 & $1.95(0.002,1728.03)$ & 0.847 \\
\hline $\begin{array}{c}\text { Dengue Ig G } \\
\text { Yes }\end{array}$ & $1.22(0.32,4.64)$ & 0.772 & $0.84(0.03,26.82)$ & 0.922 \\
\hline $\begin{array}{c}\text { Hepatomegaly } \\
\text { Yes } \\
\end{array}$ & $6.85(0.46,102.34)$ & 0.163 & $21.16(0.35,1269.16)$ & 0.144 \\
\hline $\begin{array}{c}\text { Splenomegaly } \\
\text { Yes }\end{array}$ & $4.15(0.17,101.30)$ & 0.382 & $11192.2(2.78,4.50 \mathrm{e}+07)$ & 0.028 \\
\hline $\begin{array}{l}\text { Gallbladder edema } \\
\text { Yes }\end{array}$ & $1.81(0.19,17.25)$ & 0.606 & $177.5(0.125,251445.4)$ & 0.162 \\
\hline $\begin{array}{c}\text { Ascites } \\
\text { Yes }\end{array}$ & $5.55(1.12,27.50)$ & 0.036 & $75.17(0.03,159403.5)$ & 0.269 \\
\hline $\begin{array}{c}\text { Pleural effusion } \\
\text { Yes }\end{array}$ & $15.00(2.36,95.22)$ & 0.004 & $5.72(0.15,217.60)$ & 0.347 \\
\hline $\begin{array}{l}\text { SGOT (AST) } \\
100-500 \mathrm{U} / \mathrm{L} \\
500-1000 \mathrm{U} / \mathrm{L} \\
>1000 \mathrm{U} / \mathrm{L}\end{array}$ & $\begin{array}{l}9.64(3.84,24.20) \\
9.54(0.44,206.28) \\
3.29(0.09,125.92)\end{array}$ & $\begin{array}{l}<0.001 \\
0.150 \\
0.521\end{array}$ & $\begin{array}{l}4.25(0.72,24.98) \\
58.28(1.54,2204.44) \\
85.56(1.45,5046.25)\end{array}$ & $\begin{array}{l}0.109 \\
0.028 \\
0.032 \\
\end{array}$ \\
\hline $\begin{array}{l}\text { SGPT (ALT) } \\
100-500 \mathrm{U} / \mathrm{L} \\
500-1000 \mathrm{U} / \mathrm{L} \\
>1000 \mathrm{U} / \mathrm{L}\end{array}$ & $\begin{array}{l}35.75(3.52,362.58) \\
1.07(0.02,44.77) \\
0.19(0.003,10.59)\end{array}$ & $\begin{array}{l}0.002 \\
0.970 \\
0.418\end{array}$ & $\begin{array}{ll}3.06 & (0.11,82.61) \\
0.52 & (0.01,31.84) \\
0.19 & (0.002,14.63)\end{array}$ & $\begin{array}{l}0.506 \\
0.752 \\
0.453\end{array}$ \\
\hline $\begin{array}{l}\text { Lipase } \\
\text { High }(>300 \mathrm{U} / \mathrm{L})\end{array}$ & $14.15(1.45,137.41)$ & 0.022 & $35.21(3.23,383.64)$ & 0.003 \\
\hline
\end{tabular}


The relative odds of being in group B rather than in group A for having ascites was significantly 5.55 (ROR:5.55, 95\% CI: $(1.12,27.50)$; $\mathrm{p}$ value $=0.036)$ times or $(5.55-1) * 100 \%=455 \%$ higher than the corresponding relative odds for not having ascites keeping all other covariates at fixed level.

The relative odds of being in group B rather than in group A for having pleural effusion was significantly 15.00 (ROR: $15.00,95 \%$ CI: (2.36, 95.22), p value $=0.004)$ times or $(15-1) * 100 \%=1400 \%$ higher than the corresponding relative odds for not having pleural effusion keeping all other covariates at fixed level.

The relative odds of being in group B rather than in group A for having AST (100-500 U/L) was significantly 9.64 (ROR: 9.64, 95\% CI:(3.84, 24.20); $\mathrm{p}$ value $<0.001)$ times or $(9.64-1) * 100 \%=864 \%$ higher than the corresponding relative odds for AST $(>100 \mathrm{U} / \mathrm{L})$ keeping all other covariates at fixed level.

The relative odds of being in group $\mathrm{B}$ rather than in group A for having ALT (100-500 U/L) was significantly 35.75 (ROR: 35.75, 95\% CI: (3.52, $362.58), \mathrm{p}$ value $=0.002)$ times or $(35.75-1) * 100 \%$ $=3475 \%$ higher than the corresponding relative odds for ALT ( $>100 \mathrm{U} / \mathrm{L})$ keeping all other covariates at fixed level.

The relative odds of being in group B rather than in group A for having lipase ( $>300 \mathrm{U} / \mathrm{L})$ was significantly 14.15 (ROR: 14.15, 95\% CI: (1.45, $137.41), \mathrm{p}$ value $=0.022)$ times or $(14.15-1) * 100 \%$ $=1315 \%$ higher than the corresponding relative odds for lipase $(<300 \mathrm{U} / \mathrm{L})$ keeping all other covariates at fixed level.

\section{Comparison of group $C$ relative to group $A$}

For multinomial logistic relative odds ratio (ROR) estimate of group $\mathrm{C}$ relative to group A dengue patients, clinical symptoms followed by rash (ROR: 359.41 95\%CI: $(1.98,65072.0) ; \mathrm{p}$ value $=0.027)$, bradycardia (ROR: $31118.2,95 \%$ CI: $(2.04,4.75 \mathrm{e}+08) ; \mathrm{p}$ value $=0.035)$, splenomegaly (ROR: $11192.2,95 \%$ CI: $(2.78,4.50 \mathrm{e}+07)$; p value $=$ 0.028), AST 500-1000 U/L (ROR: 58.28, 95\% CI: $(1.54,2204.44) ; \mathrm{p}$ value $=0.028), \mathrm{AST}>1000 \mathrm{U} / \mathrm{L}$ (ROR: 85.56, 95\% CI: (1.45, 5046.25); p value $=0.032)$, lipase $(>300 \mathrm{U} / \mathrm{L})(\mathrm{ROR}: 35.21,95 \% \mathrm{CI}$ : $(3.23,383.64) ; \mathrm{p}$-value $=0.003)$ were statistically significant and can be interpreted like before group B relative to group A (Table 3).

\section{Discussion:}

In 2019, outbreak of dengue fever was unpredictable along with varieties of presentations. This study describes the clinical features, laboratory findings and radiological finding of DF. The maximum number of dengue cases in our study was seen in the months of July to November which indicated an active viral transmission during monsoon and post-monsoon period. This correlated with a study conducted by Gupta ${ }^{10}$ and UkeyP $\mathrm{P}^{11}$.

In a study done by the European Centre for Disease Prevention and Control, the case rate was ratio of 1 . Similar in males and females, with a male-to-female $14: 1{ }^{12}$ Studies done by the WHO Western Pacific region showed a male preponderance in cases reported from Asia in contrast to the studies done in South America where it has been reported in equal proportions of male and female dengue cases or a greater proportion of female cases ${ }^{12}$. In our study, the male patients slightly outnumbered the female patients and the male-to-female ratio was 1.16:1. But notably, male patients more frequently developed severe dengue $(23.7 \%)$ compared to female patients $(8.8 \%)$.

In our study, most of the patients were in the age group of 18-49 years (68.6\%). Dengue infections were observed more frequently in age group $<18$ years belonged to group $\mathrm{A}(40.6 \%)$. Most of the patients belonged in group B at the age 18- 49 years (49.0\%) and age 50 and above (70.3\%).

Typical manifestations of dengue fever are acute febrile illness with chills, body aches, myalgia, joint pain, petechial rash and bleeding manifestations. Several studies of travelers or military people have reported these "classic" symptoms of dengue fever in $15 \%-60 \%$ of patients ${ }^{13,14}$. Regarding clinical finding in our study the known common features like fever $(91.8 \%)$, vomiting $(50.2 \%)$, headache $(48.2 \%)$,were frequent among the dengue patients, these findings are similar to the findings of Jain D et $\mathrm{al}^{15}$. But myalgia $(19.6 \%)$, rash $(14.5 \%)$, retro orbital pain $(14.1 \%)$ were not so common in this outbreak. Presence of hypotension (13.2\%), narrow pulse pressure (16.4\%) and some unusual feature like abdominal pain (49.3\%), loose motion (55\%) and cough/ breathlessness (41.4\%) were predominant. Notable clinical and radiological findings were ascites and pleural effusion, respectively (53.2\%) and (51.8\%). Hepatomegaly and peri-gallbladder edema were also common which were respectively (32.3\%) 
and $(35.5 \%)^{15}$. In 2019 outbreak some patients with dengue fever also presented with splenomegaly which was about (15.1\%). Clinical manifestations of dengue infection demonstrated that patients classified with group B were more frequently presented with typical symptoms as fever, retro-orbital pain, headache, myalgia and also with atypical presentations including vomiting, loose motion, abdominal pain, cough..

Patients classified with group B \& C were more prone to develop rash, bleeding, hypotension, bradycardia, low pulse pressure.

The hallmark of DF is fever. But few of our patients were afebrile and they were elderly. These afebrile Dengue could be the future challenge for detecting early complications of DF.

A significant relationship was found between diabetes $(p=0.003)$ and hypertension $(p=0.020)$ with dengue classification group. Those were highly occurred in group B.

Rapid diagnostic test Dengue NS1 is still the choice to detect early DF. In this study, about 18 cases were NS1 negative within 5 days of febrile episode and were later diagnosed by positive Dengue IgM test. These 18 patients had features of DF with fall in platelet count.

About 40.9\% patient had Dengue -IgG positive within 7 days of onset of illness which indicated past infection. These patients undergone longer course with complications and most of them were categorized into group $\mathrm{B}$ and $\mathrm{C}$ ( $\mathrm{P}$ value was highly significant $<.001)$. So early detection of $\mathrm{IgG}$ may be helpful to predict the course of illness. These findings is near similar to a Caribbean study $(85.7 \%)$ where cases had IgG positive on admission ${ }^{16}$. Mild to moderate rise in ALT and AST was found in a larger number of cases and high rise of ALT and AST were not very uncommon. In our study anicteric hepatitis was a common association in DF in 2019. Lipase was also increased in $28.8 \%$ cases suggesting pancreatitis is not an uncommon feature in DF.

\section{Conclusion:}

Dengue epidemics are on the rise in Bangladesh for last few years. Every monsoon brings along an outbreak of dengue. The last outbreak in 2019 was struck with an unexpected death. Though DF is a known disease to us, the clinicians observed some changes in patterns of presentation, along with severity of DF. A study like ours is a step forward to detect the pattern of presentation in terms of usual and unusual features, disease course and severity. However, dengue virus serology would have helped us to correlate clinical manifestations with virus subtype. Among the findings notable was, a common finding myalgia was not so common this time. Febrile diarrhea, Pain abdomen, pleural effusion, ascites, gall bladder edema, hepatomegaly and splenomegaly were observed more common than before. Acute liver injury with elevated AST and ALT were significant. We found few afebrile DF and most of the severe cases were re infection as evidenced by early IgG positive. As the spread of dengue and dengue hemorrhagic fever is increasing with significant mortality and /morbidity, it is extremely important to consider atypical manifestations of dengue for appropriate diagnosis and treatment.

This would aid in the providing early treatment, and better care to the patients and further studies are needed to identify any specific serotype which may be responsible for the atypical manifestations.

Conflict of interest: None.

Funding: None.

\section{References:}

1. Bhatt S, Gething PW, Brady OJ, Messina JP, Farlow AW, Moyes CL, Drake JM, Brownstein JS, Hoen AG, Sankoh O, Myers MF. The global distribution and burden of dengue. Nature. 2013 Apr;496(7446):504-7. https://doi.org/ 10.1038/ nature12060, PMid:23563266 PMCid:PMC3651993

2. Health Emergency Operation Center \& Control Room, DGHS, GOB January, 2020

3. World Health Organization. Dengue haemorrhagic fever: diagnosis, treatment, prevention and control. World Health Organization; 1997.

4. World Health Organization. Comprehensive guideline for prevention and control of dengue and dengue haemorrhagic fever.Revised and expanded edition. WHO-SEARO 2011. (SEARO Technical Publication Series No. 60)

5. Vaughn DW, Green S, Kalayanarooj S, Innis BL, Nimmannitya S, Suntayakorn S, Endy TP, Raengsakulrach B, Rothman AL, Ennis FA, Nisalak A. Dengue viremia titer, antibody response pattern, and virus serotype correlate with disease severity. The Journal of infectious diseases. 2000 Jan 1;181(1):2-9. https://doi.org/10.1086/315215, PMid:10608744

6. Martina BE, Koraka P, Osterhaus AD. Dengue virus pathogenesis: an integrated view. Clinical 
microbiology reviews. 2009 Oct 1;22(4):564-81. https://doi.org/10.1128/CMR.00035-09 PMid:19822889 PMCid:PMC2772360

7. Goh BK, Tan SG. Case of dengue virus infection presenting with acute acalculouscholecystitis. Journal of gastroenterology and hepatology. 2006 May 1;21(5):923-4. https://doi.org/10.1111/ j.14401746.2006.04122.x, PMid:16704552

8. Ahmed S, Ali N, Tariq WU. Case report neurological manifestation as presenting feature in Dengue Fever. JCPSP. 2007;17(4):236-7.

9. Sharma N, Mahi S, Bhalla A, Singh V, Varma S, Ratho RK. Dengue fever related acalculouscholecystitis in a North Indian tertiary care hospital. Journal of gastroenterology and hepatology. 2006 Apr;21(4):664-7. https://doi.org/10.1111/j.14401746.2006.04295.x, PMid:16677150

10. Gupta E, Dar L, Narang P, Srivastava VK, Broor S. Serodiagnosis of dengue during an outbreak at a tertiary care hospital in Delhi. Indian J Med Res. 2005 Jan 1;121(1):36.

11. Ukey PM, Bondade SA, Paunipagar PV, Powar RM, Akulwar SL. Study of seroprevalence of dengue fever in central India. Indian journal of community medicine: official publication of Indian Association of Preventive \& Social Medicine. 2010 Oct;35(4):517. https://doi.org/10.4103/0970-0218.74366 PMid:21278875 PMCid:PMC3026133
12. Anker M, Arima Y. Male-female differences in the number of reported incident dengue fever cases in six Asian countries. Western Pacific surveillance and response journal: WPSAR. 2011 Apr;2(2):17. https://doi.org/10.5365/wpsar.2011.2.1.002 PMid:23908884 PMCid:PMC3730962

13. Sharp TW, Wallace MR, Hayes CG, Sanchez JL, DeFraites RF, Arthur RR, Thornton SA, Batchelor RA, Rozmajzl PJ, Hanson RK, Wu SJ. Dengue fever in US Troops during operation restore hope, Somalia, 1992-1993. The American journal of tropical medicine and hygiene. 1995 Jul 1;53(1):89-94. https://doi.org/10.4269/ajtmh.1995.53.89 PMid:7625541

14. Shirtcliffe P, Cameron E, Nicholson KG, Wiselka MJ. Don't forget dengue! Clinical features of dengue fever in returning travellers. Journal of the Royal College of Physicians of London. 1998 May 1;32(3):235-7.

15. Jain D, Rajput R, Pathak V, Mittal A, Jain P. Changing Trends in Clinical Presentation and Biochemical Spectrum of Dengue Fever: An Observation of a Tertiary Care Centre. Archives of Clinical Infectious Diseases. 2017 Jul 1;12(3). https://doi.org/10.5812/ archcid.62221

16. Kumar A, Nielsen AL. Trends in the patterns of IgM and IgG antibodies in febrile persons with suspected dengue in Barbados, an English-speaking Caribbean country, 2006-2013. Journal of infection and public health. 2015 Nov 1;8(6):583-92. https://doi.org/ 10.1016/j.jiph.2015.05.013, PMid:26117708 